
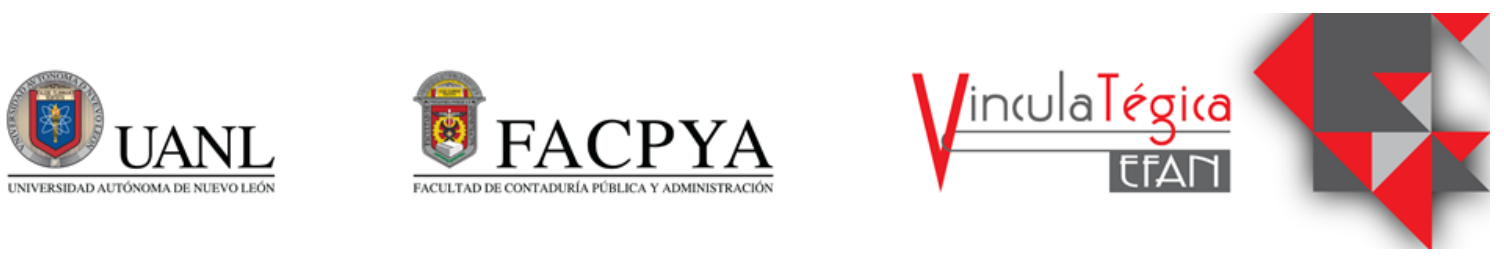

\title{
Implementación de una estrategia de marketing digital en una MiPyME del sector comercio en Campeche y su impacto en ventas
}

\author{
Fernando Medina Blum ${ }^{1}$, Román Alberto Quijano García ${ }^{2}$ y Diana Guadalupe Peña \\ Vázquez ${ }^{3}$ \\ ${ }^{1}$ Universidad Autónoma de Campeche, femedina@uacam.mx, Av. Agustín Melgar S/N Col. Buenavista C.P. \\ 24039, 9818119800 Ext. 1060101. \\ ${ }^{2}$ Universidad Autónoma de Campeche, raquijan@uacam.mx, Av. Agustín Melgar S/N Col. Buenavista C.P. \\ 24039, 9818119800 Ext. 3020100. \\ ${ }^{3}$ Universidad Autónoma de Campeche, diagpena@uacam.mx, Av. Agustín Melgar S/N Col. Buenavista C.P. \\ 24039, 9818119800 Ext. 3020100.
}

Información del artículo revisado por pares

Fecha de aceptación: junio-2021

Fecha de publicación en línea: diciembre-2021

DOI: https://doi.org/10.29105/vtga7.2-14

\section{Resumen}

El comercio electrónico es una plataforma amplia de negocio que puede ser adaptada a cualquier empresa. Actualmente la evolución de transacciones a través de este modelo de negocio ha ido en aumento en los últimos años. En México, según datos de la Asociación Mexicana de Venta Online, el comercio electrónico incrementó en 2020 un $81 \%$ en comparación al año anterior. Algunos de los beneficios del e-commerce para las empresas son la mejora de procesos organizacionales hasta la exploración de una ventaja competitiva. Es importante señalar que incursionar en este tipo de plataformas no es exclusivo para grandes empresas, las MiPyMEs pueden acceder a los beneficios del ecommerce sin necesidad de grandes inversiones, el abanico de opciones es amplio y las ventajas de implementación puede resultar muy redituable para el desarrollo de la organización. La presente investigación tiene como finalidad presentar los resultados de la implementación del comercio electrónico en una MiPyME del sector comercio, ubicada en la ciudad de San Francisco de Campeche, Campeche, México.

Palabras clave: MiPyME, Marketing Digital, ecommerce.

JEL: L81, M31.

\begin{abstract}
E-commerce is a broad business platform that can be adapted to any company. Currently, the evolution of transactions through this business model has been increasing in recent years. In Mexico, according to data from the Mexican Association of Online Sales, electronic commerce increased by $81 \%$ in 2020 compared to the previous year. Some of the benefits of e-commerce for companies are the improvement of organizational processes to the exploration of a competitive advantage. It is important to note that venturing into this type of platform is not exclusive to large companies, MiPyMEs can access the benefits of ecommerce without the need for large investments, the range of options is wide and the advantages of implementation can be very profitable for the development of the organization. The purpose of this research is to present the results of the implementation of electronic commerce in a MiPyMEs in the commerce sector, located in the city of San Francisco de Campeche, Campeche, Mexico.
\end{abstract}

Keywords: MiPyME, Marketing Digital, ecommerce.

JEL: L81, M31. 


\section{INTRODUCCIÓN}

A lo largo del desarrollo comercial de las empresas, existen momentos donde se deben enfocar los objetivos organizacionales para poder trazar el rumbo adecuado por el cual debe encaminarse las estrategias y acciones, esto incluye en ocasiones, la reestructuración de dichos objetivos para alinearse a las oportunidades de crecimiento que surgen en un mercado, que si bien en la mayoría de los casos se requiere de un conocimiento y experiencia en la implementación y operación, en otros la innovación y la decisión de realizarlo se convierte en un factor determinante no solo para la permanencia del negocio, sino también para adaptarse y explotar necesidades que el mismo mercado y la demanda que se tiene. Todo esto sin dejar de lado la esencia primordial por la cual la empresa fue creada, y que la identidad del negocio no se dañe, sino que evolucione con el pasar de los años.

Por lo anterior, el presente documento, se abordan temas que hoy en día cobran gran importancia para el desarrollo de las Micro, Pequeñas y Medianas Empresas (MiPyMEs); donde se busca tener una evidencia clara que permita tomar las decisiones óptimas para adoptar estrategias vinculadas con los modelos de negocios basados en comercio electrónico e implementarlas con una referencia cuantitativa que nos permita tener un panorama más claro de los beneficios tanto económicos y organizacionales que brindan dichas estrategias.

\section{MARCO TEÓRICO \\ 1.1. IMPORTANCIA DE LAS MIPYMES}

En México las grandes empresas solo son un pequeño porcentaje en comparación con las micro, pequeña y medianas empresas, por lo cual, se considera que son el principal motor de la economía mexicana (Filion, Cisneros y Mejía, 2011).

De acuerdo a los resultados de la Encuesta Nacional sobre Productividad y Competitividad de las Micro, Pequeñas y Medianas Empresas (ENAPROCE, 2018), realizado por el Instituto Nacional de Estadística y Geografía, en conjunto con la Secretaría de Economía, son 4.1 millones de empresas pertenecientes al sector MiPyMEs clasificadas en los sectores de manufacturas, comercio y servicios privados no financieros generando gran parte de la actividad económica y los empleos; dentro de esta clasificación las microempresas representan el 97.3\% del total de las micro, pequeñas y medianas empresas.

En cuanto a la definición de empresa Hernández y Rodríguez (2012) afirma "que es aquella entidad que requiere de recursos económicos y humanos para el logro de ciertos objetivos económicos" (p.13). Es decir, es toda aquella organización que a través de diferentes actividades provee a la sociedad de bienes y servicios y, está a su vez percibe remuneraciones económicas.

Existen diferentes criterios para clasificar a las empresas, desde el objetivo lucrativo o sin fines de lucro; si son públicas o privadas; o de acuerdo a su tamaño. No obstante, cuando se desea realizar la clasificación de acuerdo a su tamaño los criterios dependen del país u organización que lo determine.

Definir a las MiPyMEs es una tarea complicada porque no existe un criterio para los diferentes países que integran a América Latina. Argentina las define de acuerdo a su nivel de ventas que depende al sector que pertenece; Bolivia emplea tres perspectivas empleo, ventas y activos; Chile las establece de acuerdo a facturación y empleo (Saavedra y Hernández, 2018).

No obstante, otro enfoque de la definición de MiPyMEs lo ofrece el Instituto Libertad y Democracia, mencionado por Valdés y Sánchez (2012), como organizaciones que no cuentan con autorizaciones legales, por lo tanto, son empresas fuera de la legalidad, en México el $23.1 \%$ caen dentro de esta característica de acuerdo a la medición de la informalidad (INEGI, 2013).

En México, la Secretaría de Economía clasifica a las empresas de acuerdo al número de trabajadores siendo micro empresas aquellas organizaciones que, sin importar el 
sector, cuenta con hasta 10 empleados (Tabla 1). Se constituyen a partir de una oportunidad de negocio o la detección de alguna necesidad siempre con la ventaja de contar con autonomía laboral, es decir, el emprendimiento relaciona la necesidad de una persona por la generación de ingresos con el autoempleo dando como resultado una independencia económica y financiera.

Actualmente, las MiPyMEs se concentra en refrendar su consolidación, así como su éxito a partir de la competitividad, no obstante, se enfrenta a las siguientes situaciones externas (Zevallos 2005, citado por Vaca, Salinas y Vayas, 2018):

- Falta de fuentes de financiamiento externas que incentiven su crecimiento.

- Servicios públicos e Infraestructura.

- Ausencia de políticas públicas que fomenten su participación en el mercado.

- Problemas sociales que obligan al empresario a retirarse del mercado.

- Mercado interno con problemas económicos que reducen el poder adquisitivo de la población.

- Complejidad de acceso al comercio exterior.

- Falta de aplicación de tecnologías de información.

- Medio ambiente.

Las problemáticas mencionadas anteriormente no están presentes de manera conjunta en cada MiPyME, sino que dependerá de las características de su entorno. Sin embargo, retos como el financiamiento se presentan en la gran mayoría de las organizaciones. En caso de no atenderse ninguna de estas áreas las consecuencias pueden ser desfavorables para la supervivencia de este tipo de empresas.

Las empresas se enfrentan a una gran competencia en el mercado, por ello, deben mantener una ventaja competitiva frente a sus competidores. Una manera de lograrlo es a través de mantener cambios que ofrezcan novedades en productos, en procesos operativos en la industria o comercio, así como en nuevos métodos de ofrecer un servicio.
Freel (2000) mencionado por García y Galvés (2012), sostiene que las empresas pequeñas que realizan innovación obtienen crecimientos en ventas a diferencia de las que no innovan, diferentes autores coinciden en las siguientes afirmaciones:

- La innovación tiene un impacto positivo en desempeño, pero no requieren de una gran innovación para lograrlo (Yamin, 1999).

- Hsueh y Tu (2004), afirman que la habilidad para innovar tiene mayor repercusión positiva en las utilidades.

- Uno de los hallazgos más interesantes fue realizado por Bastida y García (2005), al encontrar que la innovación muestra un mayor impacto especialmente en pequeñas empresas con baja tecnología, en cambio en empresas medianas y grandes con alta tecnología la diferencia es menor.

De modo que, de acuerdo a lo anteriormente expuesto, para las microempresas trabajar en innovación les representa mayores beneficios al establecer una ventaja competitiva que les permita una mayor supervivencia en el mercado. La supervivencia de un negocio está directamente relacionada con su capacidad de obtener utilidades, más no se trata tan solo de las ganancias sino de generar ventas, porque es imposible contar con utilidades sin ventas. Por consiguiente, la primera tarea de cualquier negocio es realizar las ventas de los bienes o servicios que produzca o distribuya.

Con respecto al punto anterior, es necesario detallar el valor del cliente para la organización. Por su parte Kotler y Keller (2006), afirman que las organizaciones que colocan a la alta administración como piedra angular, se quedan atrás de las empresas que centran sus decisiones conforme a las necesidades de los consumidores.

La teoría de Rogers conjunta decisiones a nivel individual y a nivel de organización, su autor Roger (2003) citado por Jones, Alderete y Motta (2013), afirman que en las MiPyMEs las decisiones individuales son más relevantes porque es el propietario el principal administrador. 
Por esta razón, los empresarios deben enfocarse en encontrar estrategias que cumplan con la satisfacción del cliente de modo que se incrementen las posibilidades de que el mismo cliente vuelva a adquirir el bien o servicio ofrecido por la empresa. En definitiva, cualquier organización debe trabajar para obtener la lealtad de los consumidores, definiendo lealtad como el continuo compromiso de recurrir por los bienes o servicios a un mismo lugar, aunque existieran otros competidores con esfuerzos de promoción y publicidad con el fin de obtener la preferencia del consumidor (Kotler y Keller, 2006).

\subsection{Marketing Digital}

Según la Organización para la Cooperación y el Desarrollo Económicos (OCDE, 2019), incluir tecnologías digitales en los procesos productivos de las empresas genera un mayor dinamismo en sus actividades, esto a su vez genera un mayor crecimiento en las empresas existentes y contribuye al surgimiento de nuevas empresas a través de estas tecnologías de comunicación.

Las empresas nuevas con baja inversión encuentran un nicho de oportunidades al aprovechar el Internet para reducir costos operativos disminuyendo sus barreras de entrada al integrar el comercio electrónico en sus procesos de venta. Existe otro beneficio como la mejora de la comunicación con proveedores, clientes y demás interesados en el modelo de negocio.

Ahora bien, de acuerdo a la OCDE (2019), el comercio electrónico es aquella operación de compra o venta de bienes o servicios que involucra redes informáticos para la colocación del pedido, es así que para ser considerado comercio electrónico, la transacción comercial debe ser negociada a través de Internet, por lo que no es exclusivo a las características del producto negociado o el método de pago, sino que con solo el contacto vía Internet entre el comprador y el vendedor ya es considerado como comercio electrónico.

A su vez, las redes sociales son una de las fuentes de mayor ingreso en el comercio electrónico. De acuerdo con Mejía (2017), afirma que redes como Facebook venden publicidad que permite un mayor alcance a clientes potenciales, las propias empresas pueden crear perfiles para interactuar con su comunidad (clientes), o bien, obtienen retroalimentación directa a través de la continua interactividad de sus consumidores acerca de los bienes o servicios proporcionados. Para obtener resultados del uso de las redes sociales es necesario saber como promover, informar, comunicar $y$ vender productos o servicios a través de Internet, es decir, hacer uso del marketing digital (Kotler \& Keller, 2006 citado por Mejía, 2017).

Es conveniente destacar la relación que existe entre el tamaño de la organización y el uso de la plataforma Facebook como canal de ventas, ya que las empresas con dos y hasta cuatro empleados, son las que más utilizan esta herramienta, mientras que las empresas más grandes disminuyen su colocación de pedidos bajo esta modalidad (OCDE, 2019).

El impacto de los resultados del marketing digital o mercadotecnia digital no consiste únicamente en la inversión monetaria de la tecnología a implementar, sino en las diferentes estrategias usadas para establecer una fidelización de los clientes actuales o bien, atraer nuevos utilizando medios digitales.

Para lograr dicha fidelización del cliente es necesario recolectar información pertinente, con el objetivo de identificar el comportamiento de estos, y así añadir valor e ideas que incentiven su lealtad y establecer el mejor canal de comunicación para publicitar contenidos significativos y relevantes para ellos. Existen tres reglas importantes que describen la naturaleza del marketing digital (Mejía, 2017):

1. La mercadotecnia digital al realizarla correctamente se convierte en una inversión, no se debe entender como un gasto de recursos económicos o de esfuerzo.

2. El objetivo se centra en lo que se dispone a aprender y lo que se ha establecido a administrar, además de recordar que no es acerca de lo que ya se conoce.

3. El esfuerzo del marketing digital debe considerarse como un asunto colaborativo y no exclusivo a una sola persona o área, 
esto con el fin de no dejar fuera ni una idea, tampoco debe entenderse como una estrategia aislada de la planeación estratégica de la organización.

La adopción del comercio electrónico por las empresas como parte de su gestión debe seguir un proceso que permita el desarrollo de las funcionalidades de las herramientas que desea integrar en su negocio.

El modelo eMICA permite la incorporación de la estrategia de implementación del marketing digital de manera gradual, conforme la organización va desarrollando la habilidad del uso de Internet. Este modelo ofrece mayor precisión para la adopción del comercio electrónico, para ello se divide en diferentes niveles agrupados en tres etapas (Figura 1).

Cada una de las etapas posee atributos que contribuyen a identificar el nivel de adopción (Tabla 2).

La primera etapa es denominada como promoción en él la información de la empresa es detallada en una página web teniendo como objetivo compartir la imagen del negocio.

Provisión, es la segunda etapa del modelo en el cual ya existe una interacción entre cliente y empresa, de acuerdo a las características de la interacción Plana Cerpa y Bro (2006) lo dividen en:

Baja Interactividad: Se ofrece acceso a información detallada de los productos, así como una galería de imágenes a modo de catálogo.

Media Interactividad: Además de los atributos de la categoría anterior también se cuenta con ayuda en línea.

Alta Interactividad: En esta categoría se incluye servicios como mensajería a correo electrónico, chat, entre otros.

Por último, la etapa de procesamiento se caracteriza por permitir transacciones en línea es por ello que se requiere de una mayor atención a la seguridad y proveer de un perfil personal para los usuarios.

En la práctica existen varios estudios que demuestran que el uso de las redes sociales potencializa el contacto con los clientes de manera más eficiente a un menor costo que medios tradicionales. Un ejemplo de ello es el estudio realizado en Chile que concluye que las pequeñas y medianas empresas con presupuestos limitados pueden hacer uso de las redes sociales como un medio para llegar a nuevos usuarios (Claro y Correa, 2016 citado por Uribe y Sabogal, 2021).

Un estudio realizado por Aguirre y Rozo (2017), en las pequeñas y medianas empresas en Colombia arrojó que las redes sociales son las herramientas digitales utilizadas por estas empresas porque ofrecen la pauta para generar una segmentación personalizada del cliente al que se desea dirigir, mencionando que:

- Ser constante es importante para establecer un vínculo con los clientes, pero sin exceder el contenido de la organización porque existe el peligro de aburrir a la audiencia.

- No ser repetitivo en el material publicado en las redes sociales, en caso de manejar más de una plataforma social los contenidos deben ser diferentes entre sí.

En México 6 de cada 10 pequeñas y mediana empresas realizó ventas por internet en 2020 , el incremento de más del $94 \%$ obedeció al confinamiento derivado de la pandemia COVID-19 incluso empresas que no habían incursionado en el comercio electrónico concretaron sus primeras transacciones comerciales a través del Internet, este tipo de empresas mexicanas utilizan las redes sociales para un primer acercamiento digital donde comparten además de sus productos, sus valores e información de su organización (AMVO, 2020).

Uribe y Sabogal (2021), en su estudio evaluaron a través de entrevistas con propietarios de las empresas la alienación de su estrategia de mercadotecnia digital con sus planes de acción a largo plazo, hallaron que su fin de la estrategia es el incremento de las ventas en corto plazo, es importante mencionar que a pesar de no contar con un plan estratégico o bien, no hacer uso completo de las redes sociales si existió un efecto en el incremento de clientes.

Castelló (2010), afirma que las empresas que desean personalizar su mensaje, incrementar la interacción con sus 
consumidores, y mas aún, entablar una comunicación cercana con el cliente con el fin de obtener su fidelización deben recurrir a las plataformas de redes sociales como Facebook, además, menciona la importancia de utilizar sus ventajas de segmentación y alcance para convertirse en publicidad relevante para el público.

Por lo tanto, para obtener éxito en este tipo de estrategias se debe considerar:

- Evitar ser invasivo para el usuario, es decir no interrumpir su experiencia de navegación al presentar la información del producto o servicio de manera más amigable.

- Medir los resultados obtenidos de la estrategia en mercadotecnia digital.

- Realizar investigación de mercado con el objetivo de segmentar correctamente al mercado de acuerdo al estilo de vida, conducta $\mathrm{y}$ comportamiento del consumidor.

- Recordar que la información sobre los clientes es el nuevo valor estratégico que va a marcar la diferencia entre una empresa que escucha y actúa sobre las preferencias del cliente sobre otra organización que no lo hace.

- Entablar una relación mas cercana con los clientes y no realizar campañas publicitarias generalizadas.

No obstante, es primordial establecer los criterios de medida que evalúen el retorno de la inversión que permita observar la variación de las visitas, permanencias audiencia y cobertura, es decir, realizar mediciones que evalúen la estrategia aplicada (Castello, 2010).

Real, Leyva y Heredia (2014), mencionan que el uso de las redes sociales en pequeñas y medianas empresas de Sonora, México, representan un riesgo pequeño en termino de tecnología y estrategia, así como de marketing para la organización, en contraste de todos los beneficios que se pueden obtener, como por ejemplo la innovación en el producto al estar en constante contacto con las necesidades de la demanda del mercado al hacer uso de la información recolectada por estos canales de comunicación. De ahí la importancia de establecer una estrategia de marketing digital para incrementar la competitividad, posicionarse en el mercado y perdurar en la preferencia de los clientes.

\section{MÉTODO}

\section{Antecedentes de la organización}

La empresa tomada como caso de estudio es una MiPyME del sector comercial ubicada en Campeche, México. Actualmente no cuenta con una metodología enfocada en la implementación de estrategias de ventas que permitan su desarrollo y rentabilidad. Por tal motivo se buscó tener una referencia base que ayude a ubicar a la empresa dentro del desarrollo e implementación del modelo eMICA; es por esto que se utilizó la escala de Likert con 5 niveles de ítem para evaluar correctamente el grado de adopción del modelo eMICA para esta organización, siendo 5 el mayor nivel de adopción del atributo y 0 en el caso de ausencia de este mismo. Luego de revisar los resultados arrojados (Tabla 3, Tabla 4 y Tabla 5) se puede determinar como diagnóstico que esta MiPyME sólo cumple con el nivel 1 de provisión dentro del modelo eMICA. Posteriormente a dicho diagnóstico se puso en marcha la adquisición de evidencia que sustente la efectividad de implementar una metodología enfocada en el marketing digital, para así lograr continuar el desarrollo de dicho modelo que permita adaptarla al negocio y lograr un posicionamiento en el mercado y fidelización entre los clientes.

\section{Planteamiento del problema}

La empresa utiliza herramientas y estrategias comerciales sin una planeación estratégica formal tales como: promoción, publicidad e implementación de las tecnologías de la información y la comunicación disponibles en la actualidad. Al no contar con esta planeación, le resulta muy difícil captar nuevos clientes y mantener los clientes actuales a través de estas herramientas de negocios, visualizar las tendencias y preferencias que puedan servir para realizar análisis que brinden información oportuna para sustentar tomas de decisiones, y con ello, lograr una ventaja competitiva mediante la automatización de procesos de recolección de datos de consumo.

El diagnóstico arrojado al momento de apegar 
esta MiPyME del sector comercial a la metodología de implementación del modelo eMICA, contempla demostrar la viabilidad de integrar una estrategia mediante el uso de medios electrónicos que ayude a potencializar las ventas, es por ello, que se necesita comparar los registros de ventas, antes y después de la implementación de dicha estrategia de negocios.

\section{Objetivo}

Obtener evidencia cuantitativa mediante la comprobación de una prueba de hipótesis que compare dos muestras tomadas en momentos específicos; para lograr demostrar el cambio significativo donde se refleje la presencia y la ausencia de una estrategia usando medios electrónicos, una vez implementada la estrategia basada en el modelo eMICA.

\section{Metodología de la investigación}

En esta investigación se realiza para obtener información cuantitativa que pueda demostrar la necesidad y la eficacia de implementar una metodología que sirva como base para el planteamiento de estrategias de negocio.

Para esto se revisaron los registros históricos contables de operación en la empresa, que pudieran apoyar a la determinación de la existencia de una diferencia significativa entre los montos de ventas sin la implementación de alguna estrategia comercial documentada y los montos de ventas durante la implementación de una metodología enfocada al marketing digital. De acuerdo a lo anterior, para el objeto de estudio solo fueron consideradas los registros de ventas antes de la implementación de la estrategia y posterior a la implementación de medios electrónicos para el desarrollo comercial.

Una vez identificado el objeto de estudio se requirió utilizar una prueba estadística que permita obtener evidencia necesaria para determinar el desempeño de la estrategia implementada. Para esto se recurrió a los datos históricos contables disponibles, donde se pudieron recolectar registros de ventas antes de la implementación de alguna estrategia (Tabla 6) y registros de ventas realizadas implementando medios electrónicos para efectuar dicha transacción (Tabla 7).

Estableciendo como base la situación anterior y tomando en cuenta que ambas muestras tienen menos de 30 datos, lo cual estadísticamente se consideran muestras pequeñas, se requirió una fórmula para determinar una comparación entre muestras pequeñas cuando sus varianzas poblacionales no se conocen, dado que no existe alguna medición histórica anterior y asumiendo que no hay diferencia significativa entre dichas muestras; esto significa que se utilizará una prueba de hipótesis para medias con varianzas poblacionales desconocidas y asumiendo igualdad entre estas muestras, por lo cual se implementó la siguiente fórmula (Myers, Myers y Ye, 2012, p. 344):

$$
t=\frac{\left(\overline{x_{1}}-\overline{x_{2}}\right)-d_{0}}{S_{p} \sqrt{\frac{1}{n_{1}}+\frac{1}{n_{2}}}}
$$

Donde:

$\mathrm{X}_{1}=$ Promedio de ventas aplicando estrategia de marketing digital.

$\mathrm{X}_{2}=$ Promedio de ventas antes de aplicar la estrategia.

$\mathrm{d}_{0}=$ Diferencia entre ventas históricas .

$S_{p} \sqrt{\frac{1}{n_{1}}+\frac{1}{n_{2}}}=$ Error muestral aproximado.

$\mathrm{n}_{1}=$ Tamaño de muestra para ventas aplicando estrategia de marketing digital.

$\mathrm{n}_{2}=$ Tamaño de muestra para ventas antes de aplicar la estrategia.

$S_{p}=\sqrt{\frac{\left(n_{1}-1 * S_{1}^{2}\right)+\left(n_{2}-1 * S_{2}^{2}\right)}{n_{1}+n_{2}-2}}$

$S_{1}^{2}=$ Varianza aproximada de ventas aplicando estrategia marketing digital.

$S_{2}^{2}=$ Varianza aproximada de ventas antes de aplicar la estrategia.

Una vez determinado la fórmula que nos apoyará para realizar la prueba de hipótesis, se procedió a plantear dichas hipótesis las cuales serán sujetas a comprobación; mismas que quedaron formuladas y descritas en la Tabla 8.

Al observar el planteamiento de las hipótesis, las cuales se buscan someter a 
comprobación y que es el objetivo medular de esta investigación, se reconoció que la hipótesis nula $\left(\mathrm{H}_{0}\right)$ parte de lo que se menciona anteriormente donde la suposición es que el promedio de ventas aplicando estrategia de marketing digital $\left(\overline{\mathrm{X}}_{1}\right)$, no presenta una diferencia significativa al promedio de ventas antes de aplicar la estrategia $\left(\overline{\mathrm{X}}_{2}\right)$; lo cual si resultara aprobada no tendría caso implementar alguna estrategia de marketing digital, por tal motivo en la hipótesis alternativa $\left(\mathrm{H}_{1}\right)$ se plantea el escenario contrario donde en caso de ser rechazada la hipótesis nula $\left(\mathrm{H}_{0}\right)$; se tendría que aceptar que efectivamente existe una diferencia entre estos montos de ventas.

Derivado del planteamiento de las hipótesis, se observa que la prueba para el estadístico de muestra (t) será comprobada en dos extremos o bien una prueba de hipótesis a dos colas; esto quiere decir que si existiera evidencia comprobable de la diferencia entre estos montos de ventas, se podría conocer cuál es el momento predominante ya sea antes o después de aplicar dichas estrategias de marketing digital; puesto que dependiendo de donde se establezca el estadístico de muestra (t) (Tabla 9), se obtendrá una referencia estadística más certera, por esta razón, esta prueba se le aplicará un nivel de significancia $(\alpha)$ de 0.05 , lo que quiere decir que se tendrá un nivel de confianza del $95 \%$, ya que la prueba es de dos colas; por lo cual, los puntos críticos ayudarán a determinar si la hipótesis nula $\left(\mathrm{H}_{0}\right)$ es aceptada o rechazada, quedarían en \pm 2.0484 ya que los grados de libertad ponderados o conjuntos $\left(n_{1}+n_{2}-2\right)$ que resultó para la prueba de hipótesis es 28.

\section{RESULTADOS}

Al momento de calcular los resultados, se obtuvo un promedio de ventas mensuales aplicando estrategia de marketing digital $\left(\mathrm{X}_{1}\right)$ de $\$ 13,502.00$ M.N. con una desviación estándar aproximada $\left(S_{1}\right)$ de $\$ 6,628.56$ M.N., mientras que para el promedio de ventas mensuales antes de aplicar la estrategia $\left(\mathrm{X}_{2}\right)$ fue de $\$ 3,300.00$ M.N con una desviación estándar aproximada $\left(S_{2}\right)$ de \$1,285.04 M.N.; esta información fue necesaria para poder convertir ambas desviaciones en un error muestral aproximado $\left(S_{p} \sqrt{\frac{1}{n_{1}}+\frac{1}{n_{2}}}\right)$, mismo que arrojó un valor de \$1,512.12 M.N.

A partir de la información anterior e implementando el modelo estadístico de la prueba de hipótesis para medias con varianzas poblacionales desconocidas y asumiendo igualdad entre las muestras, se obtuvo un estadístico de muestra (t) de 6.7468 el cual comparándolo con los puntos críticos $( \pm 2.0484)$ quedaría en zona de rechazo, puesto que dicho estadístico de muestra (t) cae fuera y se aleja en gran medida de los límites establecidos; lo que deja una evidencia concreta que con un nivel de significancia $(\alpha)$ de 0.05 se requiere rechazar la hipótesis nula $\left(\mathrm{H}_{0}\right)$ y aceptar la hipótesis alternativa $\left(\mathrm{H}_{1}\right)$, la cual propone que efectivamente existe una diferencia significativa entre los promedios de ventas mensuales en ambas muestras.

\section{CONCLUSIONES}

$\mathrm{Al}$ calcular los promedios de ventas mensuales en ambos momentos, antes y después de la implementación de la estrategia, resulta evidente una mejoría en las ventas después de implementar una estrategia de marketing digital para la MiPyME propuesta como caso de estudio.

Sin embargo, para contar con una certeza validada mediante un método estadístico de prueba de hipótesis, se procedió a evaluar la información arrojada para ambas muestras; obteniendo como resultado que efectivamente existe una diferencia significativa del desempeño en los montos de ventas mensuales, ya que el valor esperado del estadístico de muestra (t) debió caer dentro de los puntos críticos $( \pm 2.0484)$ como lo planteaba la hipótesis nula $\left(\mathrm{H}_{0}\right)$, y al ser comparado con el resultado obtenido de 6.7468 se infiere un aumento de por lo menos tres veces más en la efectividad de la estrategia de venta.

Además al ser un resultado positivo queda como evidencia que el promedio de ventas aplicando estrategia de marketing digital $\left(\bar{X}_{1}\right)$ es mayor que el promedio de ventas mensuales antes de aplicar la estrategia $\left(\mathrm{X}_{2}\right)$; esto permite corroborar que entre ambas 
muestras existe un diferenciador, y se puede establecer con un nivel de significancia $(\alpha)$ de 0.05 , que el desarrollo de este diferenciador es dictado por la implementación de la estrategia de marketing digital.

Ahora bien, el éxito de esta estrategia no debe ser considerado como consecuencia de un plan aislado con efectos inmediatos a corto plazo, sino que debe alinearse a la estructura de la planeación estratégica en la organización e identificar las diferentes herramientas que ofrece el comercio electrónico (e-commerce) para ser implementados y adaptados a las necesidades de desarrollo de las MiPyMEs.

Asimismo, se debe tomar en cuenta que el pilar del comercio electrónico es la experiencia del usuario, porque a partir de ello, dará como resultado el tipo de interacción que tendrá el cliente con la empresa, por lo que es necesario, mantener una calidad operativa en actividades como la publicidad, descripción del producto, método de pago, interfaz, seguridad, entre otras, que garanticen no solo una permanencia en el mercado, sino que se pueda identificar una ventaja competitiva que coadyuve a la fidelización del cliente, así como también para que sean considerados como elementos sustanciales de los procesos de evaluación de las estrategias comerciales. 


\section{REFERENCIAS}

Aguirre, J. C., y Rozo, J. D. (2017). Marketing digital en las pymes de Bogotá [tesis de grado]. Colegio de Estudios Superiores de Administración. https://repository.cesa.edu.co/bitstream/handle/10726/1816/TG_817.pdf?sequence=1\&isAllowed=y

Asociación Mexicana de Venta Online (2020). Estudio sobre Venta Online en PyMEs 2020. $2^{a}$ Edición, https://www.amvo.org.mx/estudios/estudio-sobre-venta-online-en-mexico-2020/

Cerpa, Narciso, Ruiz-Tagle, Andrés, Cabrera, Carolina, Hadweh, Pamela, \& Vergara, Fabián. (2007). Evaluación del nivel de adopción de internet en las universidades chilenas en base al modelo eMICA. Ingeniare. Revista chilena de ingeniería, 15(3), 270-282. https://dx.doi.org/10.4067/S0718-33052007000300007

Diario Oficial de la Federación [Secretaría de Economía]. Acuerdo por el que se establece la estratificación de las micro, pequeñas y medianas empresas. 30 de junio de 2009.

García Pérez De Lema, D., y Gálvez Albarracín, E. J. (2012). Impacto de la innovación sobre el rendimiento de la MIPYME: Un estudio empírico en Colombia. Estudios gerenciales, 28(122).

Hernández y Rodríguez, S. J. (2012). Introducción a la administración: teoría general administrativa: origen, evolución y vanguardia (Quinta edición). McGraw-Hill Interamericana editores.

Instituto Nacional de Estadística y Geografía, (2018). Encuesta Nacional sobre Competitividad de las Micro, Pequeñas y Medianas Empresas. México: INEGI http://wwwinegi.org.mx/

Instituto Nacional de Estadística y Geografía, (2019). Medición de la informalidad. México: INEGI. https://www.inegi.org.mx/temas/pibmed/

Jones, C., Alderete, M., y Motta, J. (2013). Adopción del comercio electrónico en Micro, Pequeñas y Medianas empresas comerciales y de servicios de Córdoba, Argentina. Cuadernos de Administración, 29(50),164-175.[fecha de Consulta 28 de Abril de 2021]. ISSN: 0120-4645. https://www.redalyc.org/articulo.oa?id=225029797006

Filion, L., Cisneros, L., y Mejía-Morelos, J. (2011). Administración de PYMES. Recuperado el 7 de diciembre de 2019, de http://cpx.mx/acabrera/bStarter/Administracion_de_PYMES.pdf.

Kotler, P. y Keller, K. (14 Ed.). (2012). Dirección de Marketing. Pearson Education.

Mejía Trejo, J. (2017). Mercadotecnia digital: una descripción de las herramientas que apoyan la planeación estratégica de toda innovación de campaña web. Grupo Editorial Patria. https://elibro.net/es/ereader/uacam/40496?page $=58$

Walpole, R., Myers, R., Myers S. y Ye, K. (9 Ed.). (2012). Probabilidad y estadística para ingeniería y ciencias. Pearson Education.

Organización para la Cooperación y el Desarrollo Económico, (2019). Panorama del comercio electrónico. Políticas, Tendencias y Modelos de Negocio. Editorial OECD, https://doi.org/10.1787/23561431

Organización para la Cooperación y el Desarrollo Económico, (2017). Perspectivas de la OCDE sobre la economía digital 2015. Editorial OECD, https://www.oecd.org/sti/ieconomy/DigitalEconomyOutlook2015_SP_WEB.pdf

Uribe Beltrán, Clara Inés, y Sabogal Neira, Daniel Fernando (2021). Marketing digital en micro y pequeñas empresas de publicidad de Bogotá. Universidad \& Empresa, 23(40), .[fecha de Consulta 25 de Abril de 2021]. ISSN: 0124-4639. Disponible en: https://www.redalyc.org/articulo.oa?id=187265084005

Vaca, J. E. J., Salinas, J. M. G., y Vayas, C. V. M. (2018). Las habilidades directivas en el manejo de las finanzas para la Pequeña y Mediana Empresa. Revista Publicando, $5(14$ (2)), 214-223.

Valdés, J., y Sánchez, G. (2012). Las MiPyMEs en el contexto mundial: sus particularidades en méxico. Revista de Ciencias Sociales de la Universidad Iberoamericana, VII(14),126-156.

https://www.redalyc.org/articulo.oa?id=211026873005 


\section{ANEXOS}

Tabla 1. Estratificación de las micro, pequeñas y medianas empresas.

\begin{tabular}{|c|c|c|c|c|}
\hline \multicolumn{5}{|c|}{ Estratificación } \\
\hline Tamaño & Sector & $\begin{array}{l}\text { Rango de } \\
\text { número de } \\
\text { trabajadores }\end{array}$ & $\begin{array}{l}\text { Rango de monto de } \\
\text { ventas anuales } \\
\text { (mdp) }\end{array}$ & $\begin{array}{l}\text { Tope máximo } \\
\text { combinado* }\end{array}$ \\
\hline Micro & Todas & Hasta 10 & Hasta $\$ 4$ & 4.6 \\
\hline \multirow[t]{2}{*}{ Pequeña } & Comercio & $\begin{array}{c}\text { Desde } 11 \text { hasta } \\
30\end{array}$ & $\begin{array}{c}\text { Desde } \$ 4.01 \text { hasta } \\
\$ 100\end{array}$ & 93 \\
\hline & Industria y Servicios & $\begin{array}{c}\text { Desde } 11 \text { hasta } \\
50\end{array}$ & $\begin{array}{c}\text { Desde } \$ 4.01 \text { hasta } \\
\$ 100\end{array}$ & 95 \\
\hline \multirow[t]{3}{*}{ Mediana } & Comercio & $\begin{array}{c}\text { Desde } 31 \text { hasta } \\
100\end{array}$ & \multirow{2}{*}{$\begin{array}{c}\text { Desde } \$ 100.01 \text { hasta } \\
\$ 250\end{array}$} & \multirow{2}{*}{235} \\
\hline & Servicios & $\begin{array}{c}\text { Desde } 51 \text { hasta } \\
100\end{array}$ & & \\
\hline & Industria & $\begin{array}{l}\text { Desde } 51 \text { hasta } \\
250\end{array}$ & $\begin{array}{c}\text { Desde } \$ 100.01 \text { hasta } \\
\$ 250\end{array}$ & 250 \\
\hline
\end{tabular}

Fuente: (Diario Oficial de la Federación, 2009)

Figura 1. Modelo eMICA.

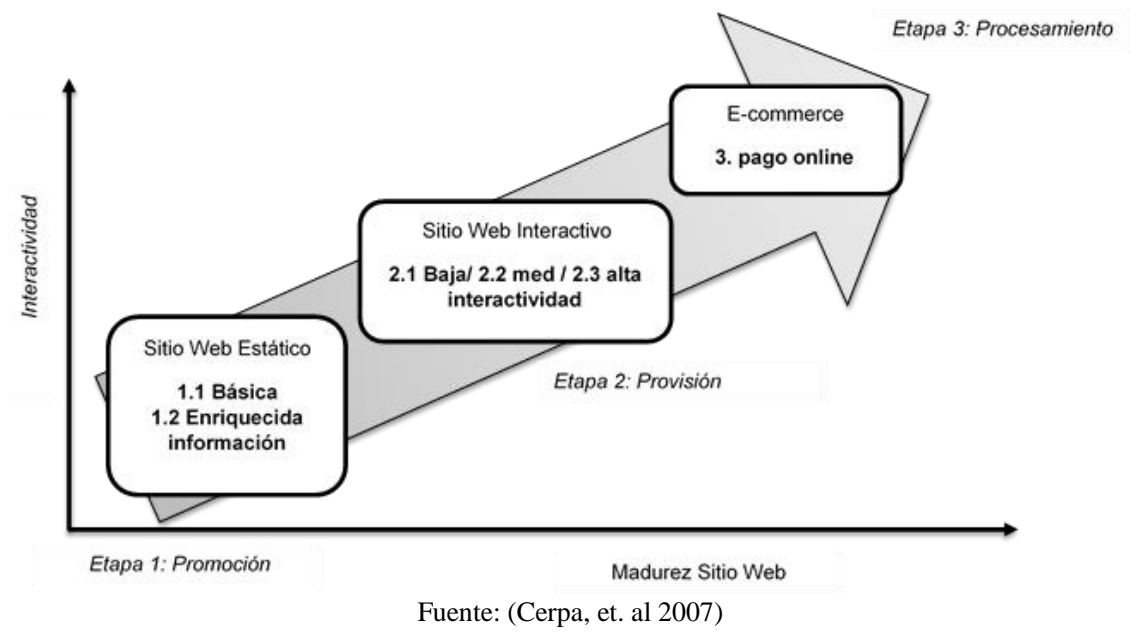


Tabla 2. Etapas y atributos del modelo eMICA.

\begin{tabular}{|c|c|c|}
\hline Etapa & Nivel & Atributos \\
\hline \multirow{6}{*}{ Promoción } & \multirow{4}{*}{ Nivel 1} & Nombre de la empresa \\
\hline & & Dirección Física \\
\hline & & Detalles de Contacto \\
\hline & & Área de Negocios \\
\hline & \multirow{2}{*}{ Nivel 2} & E-mail de Contacto \\
\hline & & Catálogo básico de servicios \\
\hline \multirow{12}{*}{ Provisión } & Nivel 1 & Dirección del Sitio Web \\
\hline & \multirow{7}{*}{ Nivel 2} & Formulario de consulta \\
\hline & & Catálogo de alto nivel \\
\hline & & Ayuda al cliente \\
\hline & & Buscadores \\
\hline & & Hiperlinks \\
\hline & & Valor agregado \\
\hline & & Mapas y rutas \\
\hline & \multirow{4}{*}{ Nivel 3} & Foro \\
\hline & & Multimedia \\
\hline & & Noticias vía mail. \\
\hline & & Opciones de lenguaje \\
\hline \multirow{3}{*}{ Procesamiento } & \multirow{3}{*}{ Nivel 1} & Transacciones Financieras \\
\hline & & Rastreo de la orden \\
\hline & & Interacción con el servidor de la empresa. \\
\hline
\end{tabular}

Fuente: (Sepúlveda, Sepúlveda y Figueroa, 2015).

Tabla 3. Diagnóstico de la etapa de Promoción del modelo eMICA de la MiPyME del Caso de Estudio, en comparación con líderes de comercio electrónico.

\begin{tabular}{llll}
\hline Etapa & Nivel & Atributos & M \\
\hline \multirow{4}{*}{ Promoción } & & Nombre de la empresa & 5 \\
& \multirow{2}{*}{ Nivel 1 } & Dirección Física & 5 \\
& & Detalles de Contacto & 5 \\
& & Área de Negocios & 5 \\
& \multirow{2}{*}{ Nivel 2 } & E-mail de Contacto & 0 \\
& & Catálogo básico de servicios & 3 \\
\hline
\end{tabular}

Fuente: (Elaboración Propia, 2019). 
Tabla 4. Diagnóstico de la etapa de Provisión del modelo eMICA de la de la MiPyME del Caso de Estudio, en comparación con líderes de comercio electrónico.

\begin{tabular}{|c|c|c|c|}
\hline Etapa & Nivel & Atributos & $\begin{array}{l}\text { Miscelánea, papelería y } \\
\text { novedades Bicentenario II }\end{array}$ \\
\hline \multirow{12}{*}{ Provisión } & \multirow[t]{3}{*}{ Nivel 1} & $\begin{array}{l}\text { Dirección del } \\
\text { Sitio Web }\end{array}$ & 0 \\
\hline & & $\begin{array}{l}\text { Formulario de } \\
\text { consulta }\end{array}$ & 0 \\
\hline & & $\begin{array}{l}\text { Catálogo de alto } \\
\text { nivel }\end{array}$ & 0 \\
\hline & \multirow[t]{7}{*}{ Nivel 2} & Ayuda al cliente & 0 \\
\hline & & Buscadores & 0 \\
\hline & & Hiperlinks & 0 \\
\hline & & Valor agregado & 0 \\
\hline & & Mapas y rutas & 0 \\
\hline & & Foro & 0 \\
\hline & & Multimedia & 2 \\
\hline & \multirow[t]{2}{*}{ Nivel 3} & Noticias vía mail. & 0 \\
\hline & & $\begin{array}{l}\text { Opciones de } \\
\text { lenguaje }\end{array}$ & 0 \\
\hline
\end{tabular}

Tabla 5. Diagnóstico de la etapa de Procesamiento del modelo eMICA de la MiPyME del Caso de Estudio, en comparación con líderes de comercio electrónico.

\begin{tabular}{llll}
\hline Etapa & \multirow{2}{*}{ Nivel } & Atributos & $\begin{array}{l}\text { Miscelánea, papelería y } \\
\text { novedades Bicentenario II }\end{array}$ \\
\hline \multirow{3}{*}{ Procesamiento } & Transacciones & 0 \\
& \multirow{2}{*}{ Nivel 1 } & Financieras & Rastreo de la orden \\
& & 0 \\
& & Interacción con el \\
servidor de la empresa. & 0 \\
\hline \multirow{3}{c}{ Fuente: (Elaboración Propia, 2019). }
\end{tabular}

Tabla 6. Datos disponibles de registros en ventas mensuales generadas antes de la implementación de alguna estrategia en la MiPyME del Caso de Estudio.

Registros de Ventas mensuales antes de aplicar una estrategia Marketing Digital. (\$ M.N.)

\begin{tabular}{lllll}
\hline 2400 & 2000 & 2600 & 2850 & 3200 \\
3050 & 2800 & 4000 & 2700 & 2600 \\
6000 & 5350 & 2500 & 2800 & 3000 \\
6500 & 2250 & 4050 & 1850 & 3500 \\
\hline \multicolumn{5}{c}{ Fuente: (Elaboración propia). }
\end{tabular}


Tabla 7 Datos recolectados de registros en ventas mensuales mediante medios electrónicos de la MiPyME del Caso de Estudio.

\begin{tabular}{ccccc}
\multicolumn{5}{c}{ Registros de Ventas mensuales Aplicando la Estrategia de Marketing } \\
Digital. (\$ M.N.) \\
\hline 12273 & 11330 & 14500 & 31640 & 10100 \\
9200 & 14000 & 9757 & 12420 & 9800 \\
\hline \multicolumn{5}{c}{ Fuente: (Elaboración propia). }
\end{tabular}

Fuente: (Elaboración propia).

Figura 2. Comparación de Ventas mensuales (\$ M.N.) entre los momentos antes y después de la implementación de la estrategia E-Commerce.

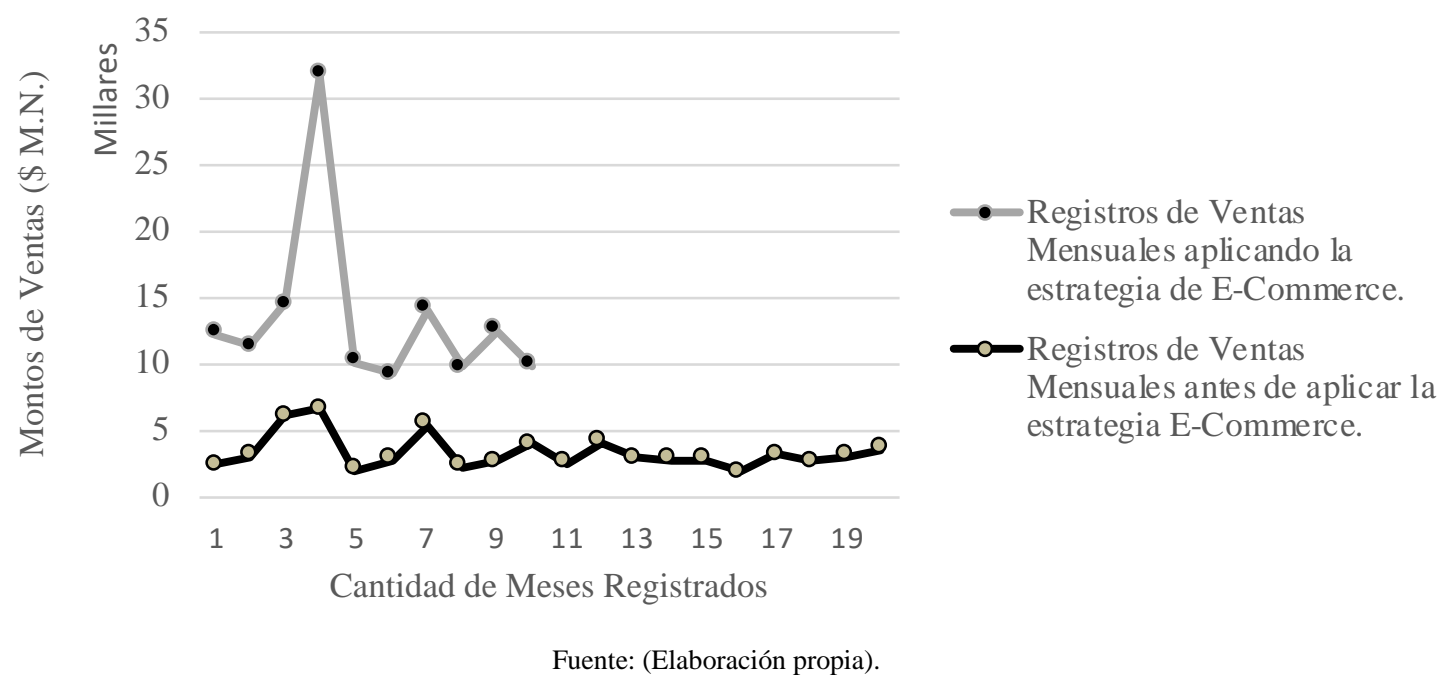

Tabla 8 Planteamiento de las hipótesis a comprobar.

\begin{tabular}{cccc}
\hline Hipótesis & Muestra 1 & $\begin{array}{c}\text { Signo de } \\
\text { Comprobación }\end{array}$ & Muestra 2 \\
\hline $\mathrm{H}_{0}:$ & $\mathrm{X}_{1}$ & $=$ & $\mathrm{X}_{2}$ \\
$\mathrm{H}_{1}:$ & $\mathrm{X}_{1}$ & $\neq$ & $\mathrm{X}_{2}$ \\
& \multicolumn{2}{c}{ Fuente: (Elaboración propia) }
\end{tabular}


Tabla 9. Valores críticos de la distribución t de Student.

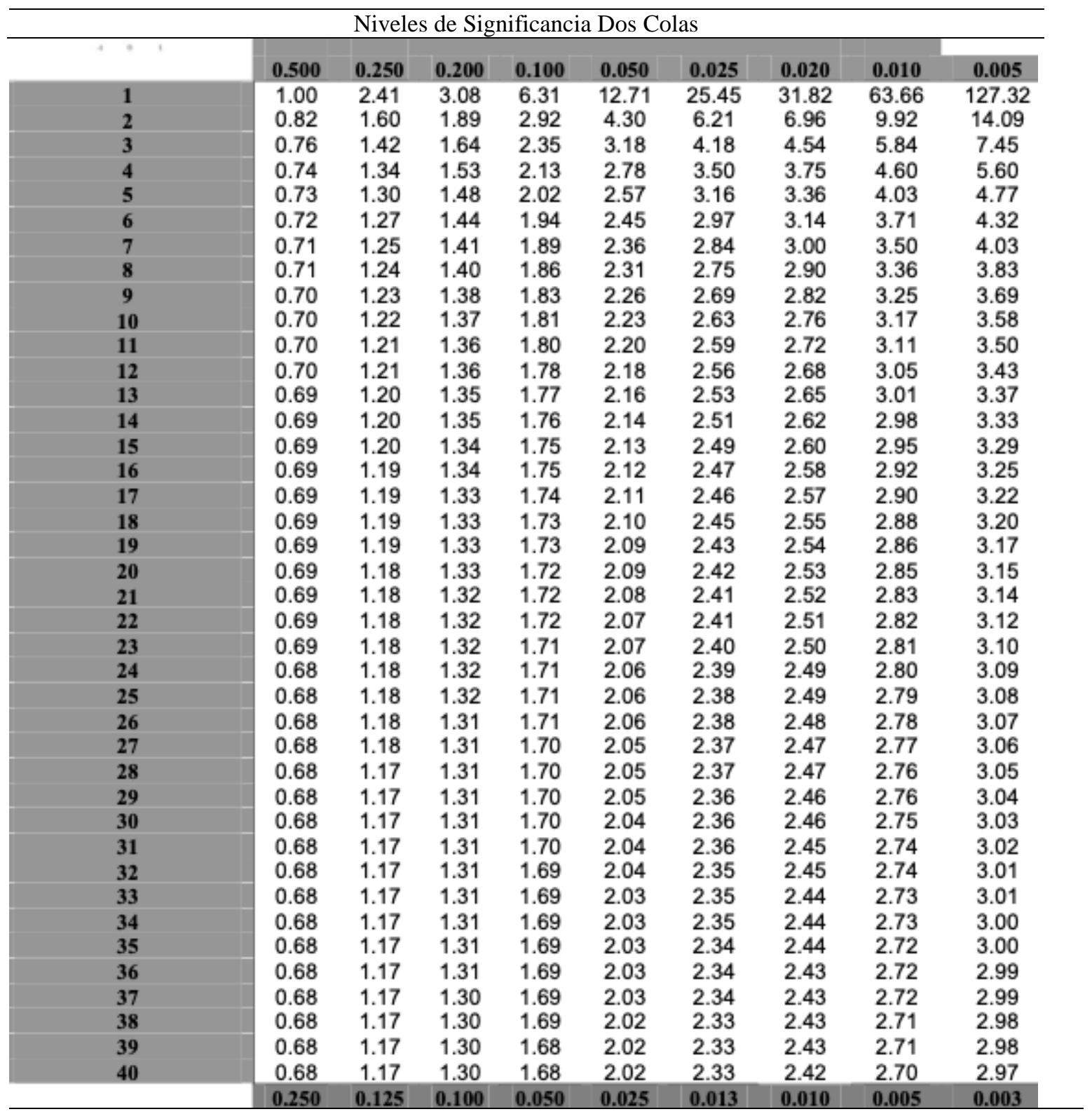

Fuente: (Myers, Myers y Ye, 2012). 